\title{
GLL
}

Geomatics, Landmanagement and Landscape No. $4 \cdot 2020,43-61$

\section{REGULAR POLYGONS IN 2D OBJECTS SHAPE DESCRIPTION}

\author{
Andrzej Kwinta, Joanna Bac-Bronowicz
}

\begin{abstract}
Summary
Many real 3D objects have complex geometric shapes in various types of analyses. Image of these objects is recorded in the form of a $2 \mathrm{D}$ map. In the analysis, a simplification of this image to basic $2 \mathrm{D}$ figures with defined geometry is often needed. The paper presents an analysis of the geometry of a flat image (an image of a 3D object) using regular polygons. Geometry properties (F form, C centroid, S size) were determined to describe the object. Various criteria of selection of the 'best' regular $\mathrm{n}$-sided polygon for a given $2 \mathrm{D}$ object (solved theoretically) were put forward. In the paper, criteria for describing a $2 \mathrm{D}$ object by regular $\mathrm{n}$-sided polygons were defined on the basis of determining the measure of object shape elongation $(e)$. In the 'blind' theoretical example, it was tested whether the individual shape measures listed in the paper allow for correct identification of the shapes of given $2 \mathrm{D}$ objects. The practical application of measures is illustrated by two actual examples. While in the first example the shape of the Canary Islands is analysed, the second example describes the shape of Poland's borders. Actual examples deliver different results for different measures. In effect, there is no clear objective criterion for selecting a polygon shape. The simplifications of the shape of an object presented in the paper should not be equated with the object's generalization. Such simplifications are used in GIS to visualize geographic analyses based on the data available in the primary database, because the object will retain the character of the shape in the simplest possible geometry and neighborhood, and does not lose any of the scope and accuracy of the attributes assigned to a given object in the database.
\end{abstract}

\section{Keywords}

GIS • polygon geometry analysis $\bullet$ regular polygon • geometric object simplification

\section{Introduction}

Various fields of knowledge and technology require analyses of the geometry of real objects as well as descriptive and statistical data assigned to their images [Bertin 1983, Müller et al. 1995, Dent 1996, Liu et al. 2010] used in spatial information systems (GIS) [Harrie and Weibel 2007]. Real objects are presented on a plane in the forms of photos, sketches, plans or maps. Map generalization uses transformation operations to create smaller-scale maps from larger-scale maps, being a key procedure for modelling and 
understanding geographical space. In such a case, these real objects are usually transformed into their projections on a horizontal plane, and thus take a two-dimensional (often simplified) shape [Angel et al. 2010]. For the purposes of analysis, real objects are sometimes recorded using multi-representation databases as simplified (generalized) two-dimensional (2D) objects. [He et al. 2018] The 2D objects that will be analysed in this paper are polygonal lines, not intersecting, closed, and with $m$ vertices $(m>2)$, constituting a simply connected domain. This assumption simplifies the conduct of analyses and is sufficient at this stage of work on this issue.

Each object can be determined by assigning three geometric properties [Prokop Reeves 1992]. These basic geometric properties are:

- $\mathrm{F}$ - form,

- C - centroid,

- $\mathrm{S}$ - size.

Every simply connected object can be geometrically defined by FCS properties. For 2D objects, the description of form is often limited to simple geometric figures; the centroid are two coordinates of the centre of gravity in the adopted coordinate system; and the size can be identified with an area, for example. Descriptive data can refer to a figure, but it is more useful to assign qualitative or quantitative values to a centroid.

Of the many simple geometric figures, convex polygons are particularly important in the description of 2D object geometry. It is crucial to select the number of vertices for a given case of analysis. An interesting issue is determining criteria for the selection of polygon geometry for a particular real object in order to minimize the number of polygon vertices. When the number of vertices of a regular polygon tend to infinity, a description of an object is obtained using a circle (examining the circularity of an object).

The geometric properties of a polygon with which the geometry of an object is described can also be defined via FCS, whereby the properties of the polygon should correspond to the geometric properties of the described object.

The shape of an actual object is very often similar to a theoretical figure, though sometimes assigning an object to a particular shape is a very subjective decision. It has long been attempted to attribute specific shapes to different objects. Initially, it was descriptive [Kohl 1850], and only later different shape measures were being introduced [Kosturbiec 1972]. Some analyses examine the belonging of an actual object to a theoretical shape (for example, a circularity study, or a study of whether an object is a rectangle). In this regard, it is important to determine the criterion for matching a figure to an actual object. For individual objects, the analyst can decide to assign a figure. For large collections of objects that are automatically analysed, it is necessary to adopt an appropriate measure and criterion.

Depending on the purpose of object description, different criteria for matching a polygon to an object may be applied. For example, in many numerical cases, in image analysis, the smallest polygon surrounding a given real object is sought as a criterion of polygon selection for that object. Studying the shape of an object and determining its regularity is another, separate issue. For example, the circularity of an object is 
examined, whether an object is a rectangle, whether it is L-shaped, and so on [Hu 1962, Peura and Ilvarinen 1997, Bae et al. 2009, Zandonadi et al. 2013].

This paper will propose a description of the geometry of $2 \mathrm{D}$ objects using regular convex polygons. The considerations will be illustrated by three examples. The theoretical example will test the correctness of various measures as to their use for regular shape analysis (algorithm correctness). In actual examples, different measures for an analysis of real objects will be compared.

Images of objects and phenomena were made by man due to a social need of sharing geographical information. The generalisation of geographical information has been and still is a necessity resulting first from data scarcity and now from data abundance [Douglas and Peucker 1973, Brassel and Weibel 1988, Bard and Ruas 2005, Chrobak et al. 2007, Mackaness and Ruas 2007].

The development of methods of generalization (simplification) of object geometry in digital topographic cartography used to create standard cartography studies (topographic maps in an accepted scale sequence) is for the most part an improvement of algorithms with a sample assessment of the similarity of the geometry of the figures before and after the process on the basis of the data collected in databases. It is now sought to design this process in order to make the result independent of the user, i.e. to develop a standard for selecting the characteristics necessary for automatic object generalization [Chrobak 2010]. Method of object simplification described in the paper is not a generalization in the above sense.

This is a proposal for how to generalize geometric data used to construct maps in a GIS (geographic information system) environment that present thematic information generated from a database. The article does not refer to the generalization of content (attribute data) and the consequences of its application to maps showing indicators and quantitative and qualitative phenomena with decreasing map capacity [Cebrykow 2017].

\section{Geometry of $n$-polygons}

The analysis of the geometry of a real $2 \mathrm{D}$ object that is a simply connected closed figure can be carried out based on figures with clearly defined geometric parameters. The simplest figures that can be used for this are regular convex polygons. The following definition should be introduced:

Definition 1. In Euclidean space, a regular convex $(n>2) n$-angle (polygon) is a closed polygonal with $n$ vertices, all sides of which have an equal length (equilateral) and all internal angles are equal (equiangular).

In every regular polygon one can describe a circle passing through all vertices (radius of the described circle - circumradius) and inscribe a circle that is tangent to all sides of the polygon (radius of the circle inscribed - apothem).

Figure 1 shows an example of a regular polygon $(n=6)$ with its centre $(S)$, side $(b)$, middle angle $(b)$, radii of circles inscribed and described in a polygon marked. According to the markings in Figure 1, the following dependencies can be noted: 
- central angle of polygon $(\beta)$

$$
\beta=\frac{2 \pi}{n}
$$

- $\quad$ side of polygon $(b)$

$$
b=\sqrt{R_{c}^{2}-R_{a}^{2}}=2 R_{c} \sin \left(\frac{\beta}{2}\right)=2 R_{a} \operatorname{tg}\left(\frac{\beta}{2}\right)
$$

- polygon area $(A)$

$$
A=\frac{n b R_{a}}{2}=n R_{a}^{2} \operatorname{tg}\left(\frac{\beta}{2}\right)=n R_{c}^{2} \frac{\sin (\beta)}{2}
$$

given that:

$$
\begin{aligned}
& n-\text { number of vertices, } \\
& R_{c} \text { - circumradius, } \\
& R_{a} \text { - apothem. }
\end{aligned}
$$

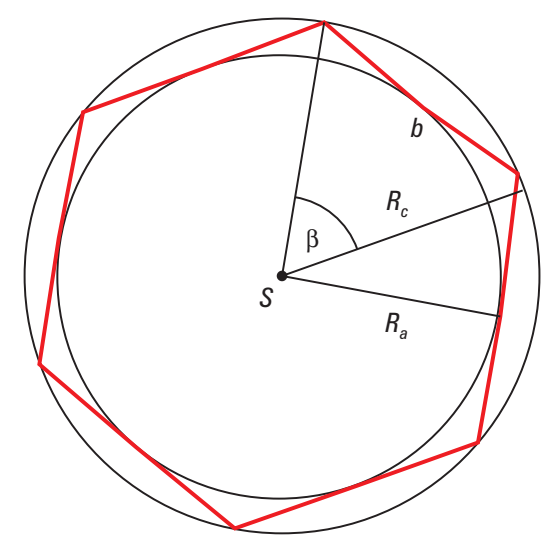

Fig. 1. Regular polygon $(n=6)$

It can be shown that the minimum distance from the central point $(S)$ of a regular polygon to the edge is apothem $\left(R_{a}\right)$, and the maximum is circumradius $\left(R_{c}\right)$.

\section{Object geometry properties}

In relation to the FCS description of the geometry of real objects, individual properties will be presented below.

The location is one of the most important properties of an object. This applies to objects of any type. Regardless of how the calculation is performed and what the adopted calculation algorithm is, the location of an object should be its invariant. Unfortunately, real objects often have very complex shapes that give rise to controversy 
as to choosing location. The basic premise is to take the right point to which an object can be assigned. Usually, it is the centroid of an object - its centre of gravity - that is adopted as this point. It is claimed here that solutions which equate average edge point values with the geometric centre of an object are wrong.

Take an actual object with $m$ vertices $\left(x_{i}, y_{i}\right)$, so it can be the following:

- object area (integral by object area)

$$
A=\int_{A} d A
$$

- centre of gravity coordinates $S\left(x_{s}, y_{S}\right)$

$$
\left\{\begin{array}{r}
x_{s}=\frac{\int_{A} x d A}{A} \\
y_{s}=\frac{\int_{A} y d A}{A}
\end{array}\right.
$$

- average coordinates $M(\bar{x}, \bar{y})$

$$
\left\{\begin{array}{l}
\bar{x}=\frac{1}{m} \sum_{i=1}^{m} x_{i} \\
\bar{y}=\frac{1}{m} \sum_{i=1}^{m} y_{i}
\end{array}\right.
$$

The equations (5) and (6) are the same for the number of edge points tending to infinity. Also, for regular objects and at evenly distributed edge points, the points $S$ (5) and $M(6)$ are the same. Unfortunately, these points are different for most real objects. It is argued that the centroid of an object should be taken as an invariant.

For the location of real objects, there is another interesting case, namely when the centroid lies outside the borders of an object (for example, for U-shaped or L-shaped objects).

The dimension of the adopted theoretical polygon depends on the purpose for which it is calculated. In literature, there are three basic criteria for choosing the size of a theoretical polygon for a real object:

- searching for the largest (largest) empty figure lying inside an object (LIP - Largest Inner Polygon) [Molano et al. 2012, Sarkar et al. 2018],

- searching for a figure with identical surface area (EMP - Equivalent Measure Polygon) [Rosin 2003, Quintet and Gniadek 2017],

- searching for the smallest (smallest) figure lying outside in which the object is located (SOP - Smallest Outer Polygon) [Smith and Chang 1996, Chaudhuri et al. 2012]. 
Of course, choosing this criterion is fundamental, and depends on the purpose for which a theoretical figure is used. The search for the largest empty figure lying inside (LIP) is related to production processes and optimization of material consumption (e.g. cutting out products from a sheet of material). In turn, the search for figures with identical surface area (EMP), but with a simpler (regular) shape is a part of generalization of objects in cartography. However, the search for the smallest surrounding figure (SOP) is very often used in image processing.

In principle, there is an unlimited variety of shapes of real objects. However, for the purpose of describing an object, the shape and name assignment of that shape to an object is evaluated. A commonly known and accepted example is a description of the shape of the Apennine peninsula, which usually is said to resemble a shoe. A very large group of irregular shapes of objects is described as letters. Therefore, L-shaped or U-shaped objects, and others.

This paper focuses on regular objects that can be described as regular polygon shapes. For this purpose, it must be defined whether an actual object can be considered regular, or whether it is possible to further suggest a similarity to a regular polygon. For example, whether the object's shape is a pentagon or is similar to a pentagon.

When analysing the shape of real objects, the numerical size of a set of elements (number of objects) is significant. For small sets, shape analysis can be conducted in one stage, while for large sets it is possible to carry out a step-by-step approach related to the geometrical properties of regular figures.

Knowing that, for regular polygons, the centre of gravity is an equidistant point from all its vertices, can be considered as the first criterion for the identification of regularity. Therefore, on the basis of this observation, the following proposition can be put.

Theorem 1. If the centre of gravity lies outside of an object or on its edge, the object is certainly not a regular convex polygon.

This proposition can be extended: if considering equations from (1) to (3), then the ratio of the radius of the circle inscribed to the radius of the circle described on this polygon can be determined for regular polygons (7):

$$
\frac{R_{a}}{R_{c}}=\sqrt{\frac{1+\cos \beta}{2}}
$$

If the possible angle values $\mathrm{b}(1)$ are taken, then the limits of possible solutions will be obtained. If, in addition, it is adopted that the radius of the inscribed circle is the minimum distance between the centre of gravity and any point on the edge of the object and the radius of the described circle corresponds to the greatest distance, then:

$$
1 \geq \frac{d_{\min }}{d_{\max }} \geq 0.5
$$

Theorem 2. If the ratio of the minimum to the maximum distance of the centre of gravity of an object from its edge is greater than 1 or less than 0.5 , the object is certainly not a regular convex polygon. 
This is an easy way to select objects that are not regular polygons. Of course, it is much more difficult to determine what kind of object is a regular polygon.

Another criterion may be used in an analysis, namely, the extended shape criterion (e) [Stojmenović and Žunić 2008]. In this regard, the appropriate inertia modules for the object as in Figure 2 need to be calculated. Axial inertia modules are (9):

$$
\begin{aligned}
& J_{x}=\int_{A} y^{2} d A \\
& J_{y}=\int_{A} x^{2} d A \\
& J_{x y}=\int_{A} x y d A
\end{aligned}
$$

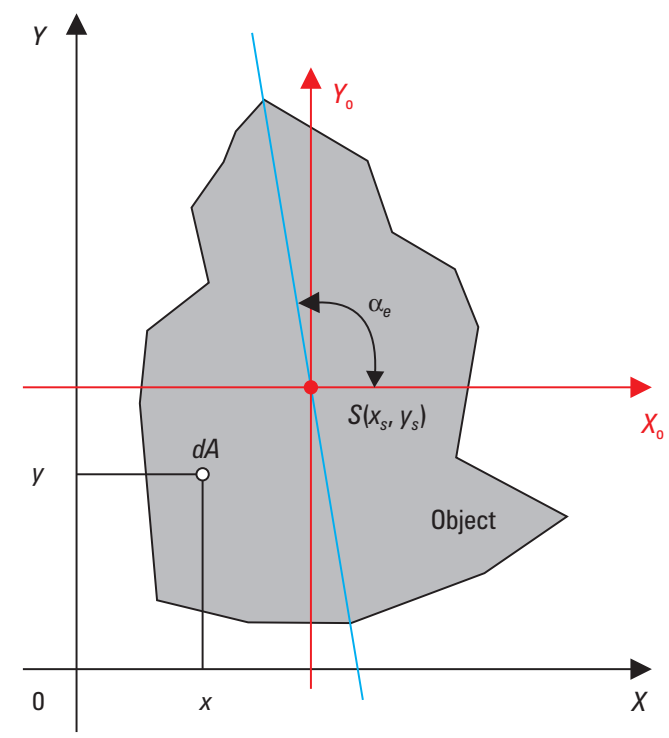

Fig. 2. Object schema for moments of inertia calculation

Figure 2 shows the direction of extension of an object that matches the direction of the maximum principal moment $\left(a_{e}\right)$. Red lines show the coordinate system axes used to calculate central moments of inertia.

Using Steiner's theorem [Pilkey 1993], central moments of inertia (Fig. 2) can be determined as follows:

$$
\begin{aligned}
& J_{0 x}=J_{x}-A x_{S}^{2} \\
& J_{0 y}=J_{y}-A y_{s}^{2} \\
& J_{0 x y}=J_{x y}-A x_{s} y_{S}
\end{aligned}
$$


Based on the equation (10), the principal moments of inertia of an object:

$$
\begin{aligned}
& J_{1}=\frac{J_{0 x}+J_{0 y}}{2}+\sqrt{\left(\frac{J_{0 x}-J_{0 y}}{2}\right)^{2}+J_{0 x y}^{2}} \\
& J_{2}=\frac{J_{0 x}+J_{0 y}}{2}-\sqrt{\left(\frac{J_{0 x}-J_{0 y}}{2}\right)^{2}+J_{0 x y}^{2}}
\end{aligned}
$$

And eventually the extension of an object as the ratio of the principal moments can be noted as:

$$
e= \begin{cases}\frac{J_{1}}{J_{2}} & J_{1} \geq J_{2} \\ \frac{J_{2}}{J_{1}} & J_{1}<J_{2}\end{cases}
$$

Analysing the shape of objects in regard to their regularity it is possible to calculate the size of their extension. Theorem 3 can be proposed as follows:

Theorem 3. For regular convex polygon the extension is equal to $e=1$.

Thus, on the basis of the above theorem each regular form elongates by 1 , while not every figure with an elongation of 1 is a regular polygon. For the analysis of real objects, the limit value of elongation $e_{1}$ should be present, for which a real object can still be thought of as a regular figure. The selection of this limit for real objects is a subjective act.

By using the $e_{1}$ limit and the centroid of an object, first, irregular objects can be selected, and then, the calculation completed. For other objects, when the elongation is less than $e_{1}$, a regular polygon shape can be determined immediately, and when it is larger, additional analysis needs to be performed.

\section{Polygon shape}

In the literature, a lot of different measures can be found to describe the shape of an object [Boyce and Clark 1964, Austin 1984, Maceachren 1985, Zunic and Rosin 2004, Loy et al. 2005, Williams and Wentz 2008]. These propositions can be grouped on theoretical basis. The following are some selected theoretical solutions for the shape index (Si) that will be tested in this paper.

Relationship between the perimeter and the area of a figure (Perimeter - Area):

$$
S i_{P}=\frac{2 \sqrt{\pi A}}{P}
$$

Relationship between the largest linear dimension and the area (Linear dimension - Area):

$$
S i_{L}=\frac{A}{L^{2}}
$$


Relationship between the area of an object and the areas of the circles inscribed and described on a figure (Area - Circle):

$$
S i_{A}=\frac{A_{W}}{A_{C}}
$$

Measure based on the values of radii from the centre of a figure to the edge (Radial):

$$
S i_{R}=\frac{100}{m \bar{R}} \sum_{i=1}^{m}\left|R_{i}-\bar{R}\right|, \quad \bar{R}=\frac{\sum_{i=1}^{m} R_{i}}{m}
$$

given that in the above equations:

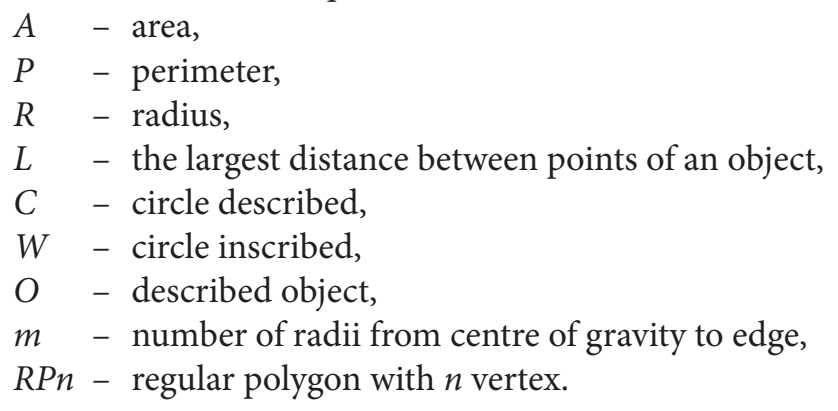

Below, Table 1 summarizes the shape measures for subsequent regular polygons based on theoretical values according to equations (13) to (16). For measure $S i_{R}$ the calculation is $m=100$ radii $R_{i}$.

Table 1. Shape measures for different regular polygons

\begin{tabular}{|l|c|c|c|c|c|c|c|c|c|}
\hline \multirow{2}{*}{ Measure } & \multicolumn{8}{|c|}{ Number of polygon vertices } \\
\cline { 2 - 10 } & $\mathbf{3}$ & $\mathbf{4}$ & $\mathbf{5}$ & $\mathbf{6}$ & $\mathbf{7}$ & $\mathbf{8}$ & $\mathbf{9}$ & $\mathbf{1 0}$ & $\infty$ \\
\hline$S i_{P}(13)$ & 0.7776 & 0.8862 & 0.9299 & 0.9523 & 0.9654 & 0.9737 & 0.9793 & 0.9833 & 1.0000 \\
\hline$S i_{L}(14)$ & 0.4330 & 0.5000 & 0.6572 & 0.6495 & 0.7197 & 0.7071 & 0.7456 & 0.7347 & 0.7854 \\
\hline$S i_{A}(15)$ & 0.2500 & 0.5000 & 0.6545 & 0.7500 & 0.8117 & 0.8536 & 0.8830 & 0.9045 & 1.0000 \\
\hline$S i_{R 100}(16)$ & 19.4012 & 9.6235 & 5.8726 & 4.0060 & 2.9609 & 2.3172 & 1.8973 & 1.6723 & 1.0000 \\
\hline
\end{tabular}

The last column corresponds to the measure values for a circle. When analysing Table 1, it is important to notice that the values of each shape measure for small amounts of polygon vertices are quite significantly different. As the number of vertices increases, the measure values come closer together. It is similar when polygons are identified by a human. Comparing a triangle with a square is simple, but comparing an octagon with a nine-angle, for instance, is troublesome. 
Since this paper is concerned with specific shapes of regular polygons, three measures based on the best matching of an object to a particular shape are analysed (Area - Shape).

$$
\begin{gathered}
S i_{S 1}=\frac{A_{O \cap F}}{A_{O \cup F}} \\
S i_{S 1}^{*}=\frac{A-A_{O \cap F}}{A} \\
S i_{S 2}=\left|\frac{A_{R P W}-A_{R P C}}{A}\right|
\end{gathered}
$$

given that:

$$
\begin{array}{ll}
A_{O} \equiv A & - \text { area, } \\
O & - \text { described object, } \\
F & - \text { polygon, } \\
R P W & - \text { polygon inscribed in a circle with a radius } R_{c} \\
R P W & - \text { polygon described on a circle with a radius } R_{a} .
\end{array}
$$

For measures (17) to (19), for each $n$ the measure values are:

$$
\begin{aligned}
& S i_{s 1}=1.0 \\
& S i_{s 1}^{*}=0.0 \\
& S i_{s 2}=0.0
\end{aligned}
$$

The measures proposed in equations (17) and (18) are equal in calculations, therefore only two measures will be further analysed.

The measures above apply to 'ideal' regular polygons. The next section provides a theoretical example of calculations for various objects in the shape of regular polygons.

For real objects, not all measures are suitable for analysis. Measure $S i_{P}(13)$ is very simple to calculate, but produces erroneous results, for example, for 'wavy' edges (changing the perimeter without changing the area). The measure $S i_{L}(14)$ is difficult for automatic analysis, because the measure limits do not belong to a single monotone sequence. Also, the measure $S i_{R}(16)$ is not conclusive for various numbers of radii (different $m$ ), because the result depends on the number of radii used in the calculation, thus it is important to always take the same number of radii. Measures based on comparisons to figures (17) to (19) are more stable, but instead are quite labour-and time-consuming (calculations can take quite a long time).

\section{Theoretical example of shape measures}

Analysis of the correctness of the adopted measures is best carried out in the form of 'blind' examples. The question was raised whether the indicated shape measures 
correctly describe the shape of objects chosen in advance. To do this, six figures were drawn, from triangle to octagon (Fig. 3), which were then digitized in the local coordinate system (saving points along the edges of an object). As is visible in Figure 3, objects resemble regular polygons in shape, but they are not exactly regular polygons.

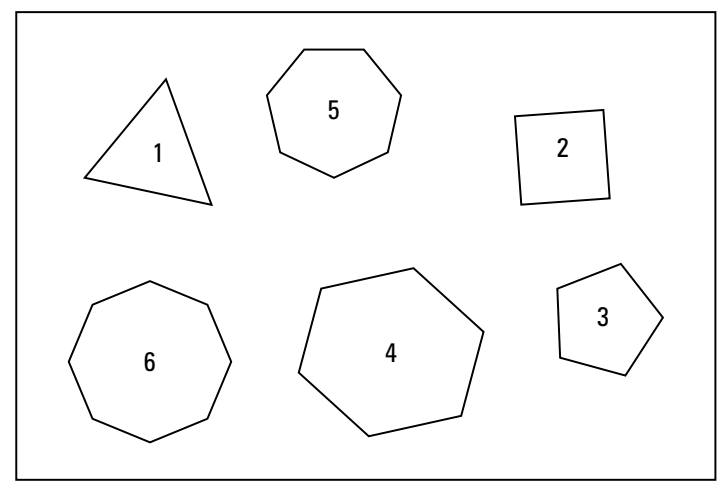

Fig. 3. Theoretical objects in measures analyses

Subsequently, using a specially developed program, a shape measure was designated for each object according to the formulas (13) to (19). The results of the calculations were divided into two tables. Table 2 shows the results for data measures calculated with equations (13) to (16). Data from Table 1 are used as reference values of the measures. The measure values obtained from the calculations were compared with the reference values, and then assigned to the resulting number of polygon vertices $n \mathrm{Si}$.

Table 2. Determination of the shape of a figure on the basis of measures $S i_{P}, S i_{L}, S i_{A}, S i_{R}$

\begin{tabular}{|c|c|c|c|c|c|c|c|c|c|c|}
\hline No. & $\begin{array}{c}\text { Theoretical } \\
\text { shape }\end{array}$ & Vertex & $S i_{P}$ & $n S i_{P}$ & $S i_{L}$ & $n S i_{L}$ & $S i_{A}$ & $n S i_{A}$ & $S i_{R}$ & $n S i_{\mathrm{R}}$ \\
\hline 1 & 3 & 18 & 0.7775 & 3 & 0.4286 & 3 & 0.2356 & 3 & 19.7292 & 3 \\
\hline 2 & 4 & 21 & 0.8879 & 4 & 0.4990 & 4 & 0.4862 & 4 & 9.5911 & 4 \\
\hline 3 & 5 & 21 & 0.9307 & 5 & 0.6481 & 5 & 0.6353 & 5 & 5.8667 & 5 \\
\hline 4 & 6 & 30 & 0.9521 & 6 & 0.6482 & 6 & 0.7428 & 6 & 4.0557 & 6 \\
\hline 5 & 7 & 23 & 0.9653 & 7 & 0.7143 & 7 & 0.7822 & 7 & 2.9443 & 7 \\
\hline 6 & 8 & 38 & 0.9745 & 8 & 0.7031 & 8 & 0.8301 & 8 & 2.4102 & 8 \\
\hline
\end{tabular}

As it is demonstrated above, in Table 2 , all measures $S i_{P}, S i_{L}, S i_{A}, S i_{R}$ correctly designated the object shapes to be analysed.

For measures based on comparative analysis with polygon shapes and searching for the extremum of a measure, the results are summarized in Table 3. Calculations were 
performed for regular polygons from $n=3$ to $n=10$. For $\mathrm{Si}_{\mathrm{S} 1}$ maximum measures are required and for $\mathrm{Si}_{\mathrm{S} 2}$ and $\mathrm{Si}_{\mathrm{S} 3}$ minimum measures. Extreme measure values for individual objects are highlighted in bold in Table 3 .

On the basis of the results obtained in Table 3 , it should be concluded that all methods correctly indicated the shapes of the analysed objects.

Table 3. Determination of the shape of a figure on the basis of measures $S i_{S 1}, S i_{S 2}$

\begin{tabular}{|c|c|c|c|c|c|c|c|c|c|}
\hline \multirow{2}{*}{$\begin{array}{c}\text { Analysed } \\
\text { shape }\end{array}$} & \multirow{2}{*}{ Method } & \multicolumn{8}{|c|}{ Regular shape } \\
\cline { 3 - 10 } & & $\mathbf{3}$ & $\mathbf{4}$ & $\mathbf{5}$ & $\mathbf{6}$ & $\mathbf{7}$ & $\mathbf{8}$ & $\mathbf{9}$ & $\mathbf{1 0}$ \\
\hline \multirow{3}{*}{3} & SiS1 & $\mathbf{0 . 9 7 8 6}$ & 0.6740 & 0.6841 & 0.7015 & 0.6854 & 0.6858 & 0.6862 & 0.6858 \\
\cline { 2 - 10 } & SiS2 & $\mathbf{0 . 0 5 8 1}$ & 0.8194 & 1.1790 & 1.3806 & 1.5047 & 1.5864 & 1.6430 & 1.6838 \\
\hline \multirow{3}{*}{4} & SiS1 & 0.6850 & $\mathbf{0 . 9 8 5 0}$ & 0.8219 & 0.8317 & 0.8324 & 0.8420 & 0.8346 & 0.8357 \\
\cline { 2 - 10 } & SiS2 & 0.6242 & $\mathbf{0 . 0 2 8 1}$ & 0.3110 & 0.4648 & 0.5582 & 0.6191 & 0.6611 & 0.6913 \\
\hline \multirow{3}{*}{5} & SiS1 & 0.6893 & 0.8243 & $\mathbf{0 . 9 8 3 3}$ & 0.8907 & 0.8952 & 0.8978 & 0.8979 & 0.9032 \\
\cline { 2 - 10 } & SiS2 & 0.8585 & 0.2321 & $\mathbf{0 . 0 2 9 9}$ & 0.1703 & 0.2550 & 0.3101 & 0.3479 & 0.3750 \\
\hline \multirow{2}{*}{6} & SiS1 & 0.7072 & 0.8300 & 0.8844 & $\mathbf{0 . 9 9 4 5}$ & 0.9197 & 0.9242 & 0.9271 & 0.9281 \\
\cline { 2 - 10 } & SiS2 & 0.9904 & 0.3756 & 0.1241 & $\mathbf{0 . 0 0 9 6}$ & 0.0899 & 0.1419 & 0.1776 & 0.2032 \\
\hline \multirow{2}{*}{7} & SiS1 & 0.6973 & 0.8350 & 0.8917 & 0.9209 & $\mathbf{0 . 9 8 4 8}$ & 0.9416 & 0.9459 & 0.9466 \\
\cline { 2 - 10 } & SiS2 & 1.0236 & 0.4178 & 0.1717 & 0.0413 & $\mathbf{0 . 0 3 6 9}$ & 0.0875 & 0.1223 & 0.1471 \\
\hline \multirow{2}{*}{8} & SiS1 & 0.6924 & 0.8413 & 0.8940 & 0.9264 & 0.9423 & $\mathbf{0 . 9 9 2 1}$ & 0.9548 & 0.9571 \\
\cline { 2 - 9 } & SiS2 & 1.0764 & 0.4715 & 0.2278 & 0.0990 & 0.0278 & $\mathbf{0 . 0 2 2 1}$ & 0.0620 & 0.0864 \\
\hline
\end{tabular}

On the basis of the results obtained in Table 3, it should be concluded that all methods based on polygon shapes correctly indicated the shapes of the analysed objects.

In Summary, all methods correctly defined the shapes of the specified test objects.

\section{Example of islands shapes analysis}

As another example, the Canary Islands archipelago was analysed whether the geometry of the individual islands of the archipelago can be determined on the basis of the proposed algorithm.

The Canary Islands belong to Spain, located west of Africa in the Atlantic Ocean. The Archipelago consists of seven main islands: El Hierro, Fuerteventura, Gran Canaria, La Gomera, Lanzarote, La Palma, Tenerife, and several smaller. The mutual distribution of islands is shown in Figure 4.

Object geometry data were obtained by digitizing a map. Table 4 contains the number of points (the column $3-k$ ) that describe the shape of the islands (after digiti- 
zation). Table 4 in column $4(A)$ shows the designated areas of the islands on the basis of digitised data.

Then, each island was analysed in section 3. For each island, the centroid, elongation and the direction of elongation were calculated, as well as the ratio of the smallest to the largest distance from the centroid (8). A critical elongation value $\left(e_{b}\right)$ equal to 1.3 was used for the calculation. The results of the calculations are summarized numerically in Table 2 and graphically in Figure 8.

Table 4. Data on analysed islands

\begin{tabular}{|c|l|c|c|c|c|c|}
\hline $\mathbf{N}$ & \multicolumn{1}{|c|}{ Island } & $k$ & $\begin{array}{c}A \\
{\left[\mathrm{~km}^{2}\right]}\end{array}$ & $\begin{array}{c}\alpha_{\mathrm{e}} \\
{[\mathrm{deg}]}\end{array}$ & $\boldsymbol{e}$ & $\frac{\boldsymbol{d}_{\min }}{\boldsymbol{d}_{\max }}$ \\
\hline 1 & El Hierro & 67 & 266.7 & 28.08 & 1.80 & 0.21 \\
\hline 2 & Fuerteventura & 204 & 1662.9 & 56.20 & 2.88 & 0.17 \\
\hline 3 & Gran Canaria & 189 & 1563.2 & 45.26 & 1.10 & 0.59 \\
\hline 4 & La Gomera & 79 & 368.5 & 161.26 & 1.27 & 0.62 \\
\hline 5 & Lanzarote & 140 & 815.1 & 34.84 & 2.66 & 0.30 \\
\hline 6 & La Palma & 93 & 713.7 & 101.01 & 1.74 & 0.36 \\
\hline 7 & Tenerife & 251 & 2039.1 & 38.79 & 1.94 & 0.23 \\
\hline
\end{tabular}

An analysis of the results of the calculations compiled in Table 4 reveals that only for two islands (Gran Canaria and La Gomera) the elongation is below 30\%. Also, the ratio of distance from centroid to the edge (8) is met for these two islands, so a full calculation was carried out for them. For the islands of Fuerteventura and Lanzarote, the elongation is large, exceeding 2.5 .

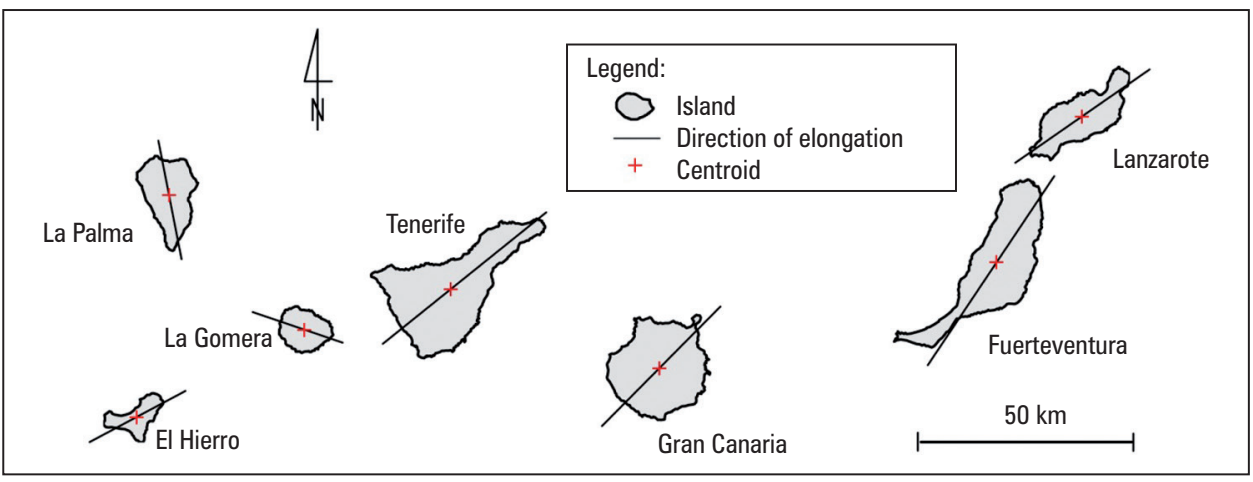

Fig. 4. Graphical presentation of calculation results 
Figure 4 shows digitized objects (islands - gray) with the calculation results. For each island, its centroid is marked as a red cross. The direction of its elongation is marked as a black line.

Table 5. Results of calculation of the shape of an island with $S i_{P}, S i_{L}, S i_{A}, S i_{R}$

\begin{tabular}{|c|c|c|c|c|}
\hline \multirow{2}{*}{ Method } & \multicolumn{2}{|c|}{ Gran Canaria } & \multicolumn{2}{c|}{ La Gomera } \\
\cline { 2 - 5 } & Measure & Shape $n$ & Measure & Shape $n$ \\
\hline$S i_{P}$ & 0.7428 & 3 & 0.8546 & 4 \\
\hline$S i_{L}$ & 0.5409 & 4 & 0.5755 & 4 \\
\hline$S i_{A}$ & 0.3444 & 3 & 0.3866 & 4 \\
\hline$S i_{R 100}$ & 53.5852 & 3 & 9.2233 & 4 \\
\hline
\end{tabular}

Table 6. Results of calculation of the shape of an island with $S i_{S 1}, S i_{S 2}$

\begin{tabular}{|c|c|c|c|c|}
\hline \multirow{2}{*}{$n$} & \multicolumn{2}{|c|}{ Gran Canaria } & \multicolumn{2}{c|}{ La Gomera } \\
\cline { 2 - 5 } & $S i_{S 1}$ & $S i_{S 2}$ & $S i_{S 1}$ & $S i_{S 2}$ \\
\hline 3 & 0.7097 & 0.5252 & 0.7390 & 0.3560 \\
\hline 4 & 0.8232 & 0.0981 & 0.8047 & 0.2276 \\
\hline 5 & 0.8933 & 0.3711 & 0.8310 & 0.4883 \\
\hline 6 & 0.8804 & 0.5201 & 0.8393 & 0.6316 \\
\hline 7 & 0.8977 & 0.6107 & 0.8354 & 0.7190 \\
\hline 8 & 0.8861 & 0.6699 & 0.8383 & 0.7763 \\
\hline 9 & 0.8886 & 0.7107 & 0.8354 & 0.8158 \\
\hline 10 & 0.8899 & 0.7400 & 0.8379 & 0.8442 \\
\hline 11 & 0.8929 & 0.7618 & 0.8357 & 0.8653 \\
\hline 12 & 0.8908 & 0.7784 & 0.8363 & 0.8814 \\
\hline
\end{tabular}

Figure 5 shows the results of the calculations of regular polygons for the two analysed islands.

When analysing the digital results in Tables 4 and 5 and graphically in Figure 5, it should be concluded that no clear results have been obtained from all calculation methods. Given the abundance of results, it could be assumed that the shape of the island of Gran Canaria is a triangle and of La Gomera is a quadrangle. However, as is shown in Figure 5, such a generalization seems wrong. Based on Figure 5, a heptagon subjectively fits 'best' to the actual shape of Gran Canaria (Fig. 5C) and hexagon to La Gomera (Fig. 5E). 

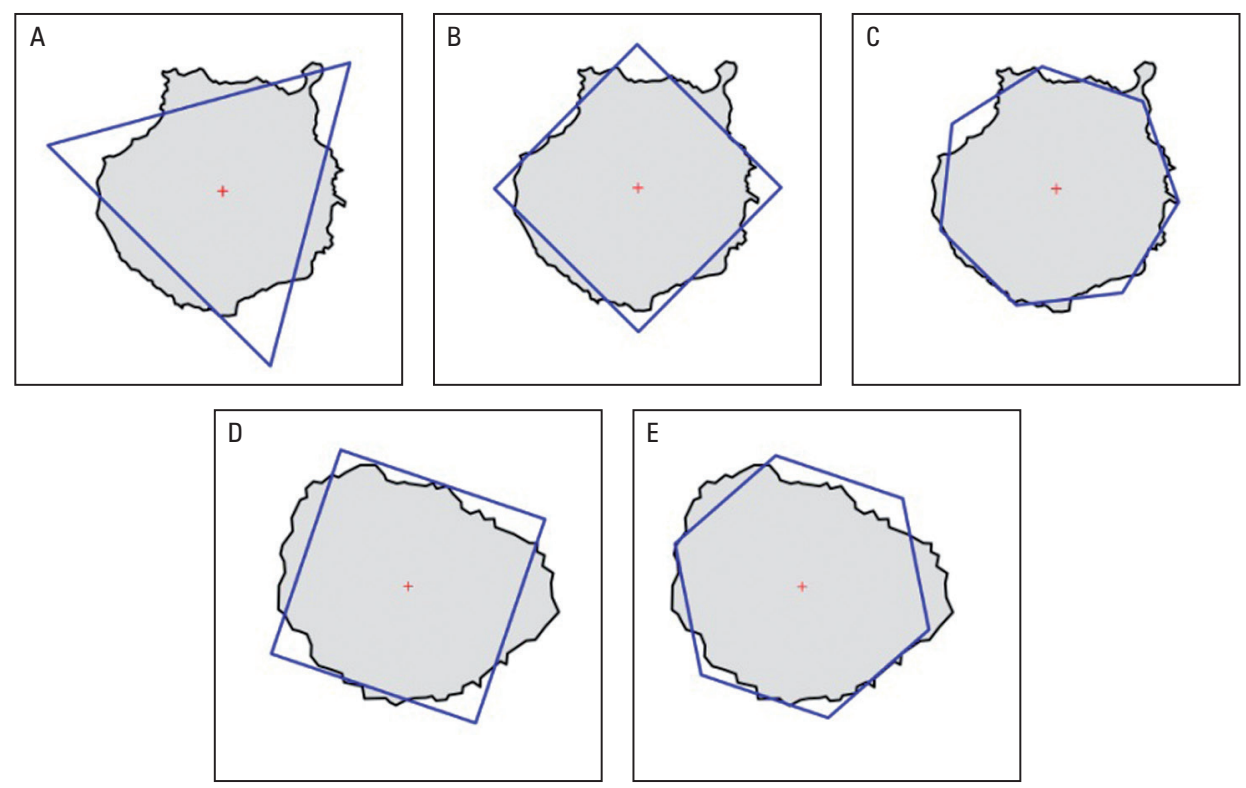

Fig. 5. Calculation results of regular polygons for the analysed cases (A, B, C for Gran Canaria; D, E for La Gomera)

As this case indicates, it is impossible to eliminate the role of editor, who must adopt the necessary degree of generalization of the shape. However, assuming that for specific statistical analyses it is necessary to adopt and maintain in the form of a map an appropriate cartographic representation, the above-described method seems appropriate to apply.

\section{Shape of country - Poland}

The second applied example, which allows to compare different criteria for choosing the shape of a regular figure, concerns the Polish borders. Based on the digitalised map, the Polish borders were obtained in local coordinate system. 160 edge points (breaks) were calculated.

Figure 6 shows the results of digitization of borders (black), centroid (red cross) and elongation direction (blue line), which are determined according to the relations shown in section 3.

The preliminary calculations gave the location (in the local coordinate system) of the centroid, the elongation $(e=1.27)$, the elongation direction $\left(\alpha_{e}=149.65 \mathrm{deg}\right)$ and the ratio of the smallest to the greatest distance from the centroid (0.55). Thus, it was indicated (on the basis of considerations in section 3) that the shape of an object is similar to a regular figure, so that searching for the 'best' polygon can be started. The shape measures in Table 7 were set out according to the formulas (13) to (19). 
As a result of the calculations, three regular polygons were obtained using different methods to describe the object (grade 3, 4, and 10 polygons).

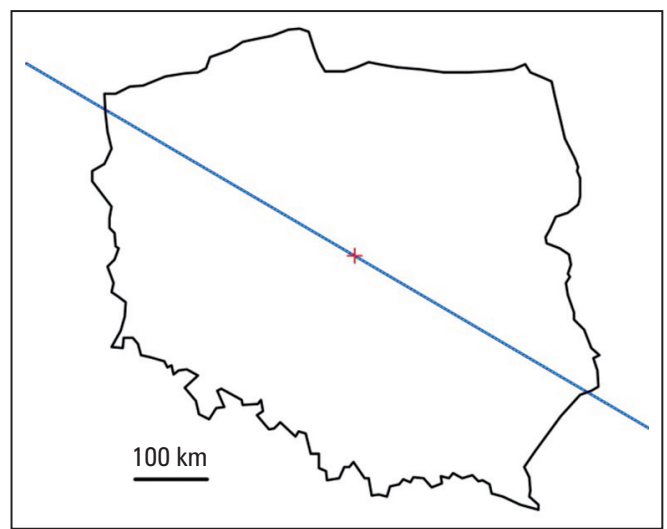

Fig. 6. Shape of Poland with marked centroid and elongation direction

Table 7. Calculation results for describing the shape of Poland with regular polygons

\begin{tabular}{|c|c|c|c|}
\hline \multicolumn{2}{|c|}{ Method } & Measure & Shape $n$ \\
\hline \multicolumn{2}{|c|}{$S i_{P}$} & 0.7278 & 3 \\
\hline \multicolumn{2}{|c|}{$S i_{L}$} & 0.4759 & 3 \\
\hline \multicolumn{2}{|c|}{$S i_{A}$} & 0.3006 & 4 \\
\hline \multicolumn{2}{|c|}{$S i_{R 100}$} & 64.7273 & 3 \\
\hline \multirow{6}{*}{$S i_{S 1}$} & 7 & 0.8163 & \multirow{6}{*}{10} \\
\hline & 8 & 0.8172 & \\
\hline & 9 & 0.8141 & \\
\hline & 10 & 0.8181 & \\
\hline & 11 & 0.8165 & \\
\hline & 12 & 0.8170 & \\
\hline \multirow{6}{*}{$S i_{S 2}$} & 3 & 0.3554 & \multirow{6}{*}{4} \\
\hline & 4 & 0.2407 & \\
\hline & 5 & 0.5073 & \\
\hline & 6 & 0.6538 & \\
\hline & 7 & 0.7433 & \\
\hline & 8 & 0.8019 & \\
\hline
\end{tabular}


Figure 7 shows the results of the analysis in graphical form from Table 7 . The shape of the analysed object (black line) with designated regular polygons (red line) is presented.

Analysis of the results indicates that different criteria for selecting regular polygons give different final results. The results are far from satisfactory, when comparing the obtained polygon shapes and the contour of Poland. In the subjective assessment of the authors of the paper, none of the obtained polygons reflects the shape of the analysed object.
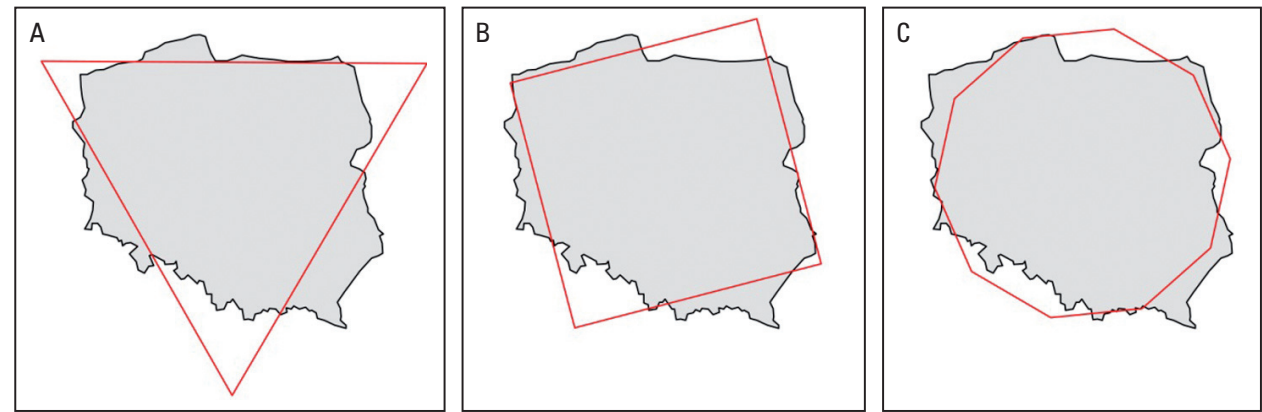

Fig. 7. Regular polygons designated for Poland's shape (A - triangle, B - quadrilateral, $\mathrm{C}$ - tenangle)

\section{Conclusions}

The aim of the paper was to examine the issue of determining the geometry of $2 \mathrm{D}$ objects using convex regular polygons. It is often necessary to replace the actual shape with a corresponding simple figure. The description of a given shape is also often used by comparison to some simple figure. Of the simple figures, special attention is given to convex regular polygons.

Introducing a uniform geometric description of an object is a very important task. The geometry of an object can be described by using properties related to its form, centroid, and size (FCS). These three FCS indicators are, of course, characterized by parameters that accurately describe the object's geometry.

The paper defines preliminary criteria that qualify objects for an analysis using regular polygons. The initial criteria are based on two solutions. The elongation of an object and the ratio of the smallest to the largest distance between the object's centroid and its edge points.

In the literature, there are many different criteria (measures) for shape description. These solutions are based on different geometric elements of objects. Several methods have been compared in the paper, and it should be stated that for objects with a shape 'similar' to regular polygons, all methods perform the calculation correctly. In the case of real objects, however, the results are less satisfactory, which means that they can be very subjective. The examples shown in the paper confirmed that for objects created 
in the basis of regular polygons the shape of objects is described by measure criteria correctly, while for real objects, the results are not satisfactory.

Further research into the description of shapes of real objects using regular polygons should be conducted in the direction of the influence of the object elongation value on the assignment of the shape.

\section{References}

Angel S., Parent J., Civco D.L. 2010. Ten compactness properties of circles: Measuring shape in geography. The Canadian Geographer, 54(4).

Austin R.F. 1984. Measuring and comparing two-dimensional shapes. In: Spatial Statistics and Models, eds. G.L. Gaile, C.J. Willmott. D. Reidel Publ. Co., Boston.

Bae S.W., Lee C., Ahn H.-K., Choi S., Chwa K.-Y. 2009. Computing minimum-area rectilinear convex hull and L-shape. Computational Geometry: Theory and Applications, 42(3).

Bard S., Ruas A. 2005. Why and How Evaluating Generalised Data? In: Developments in Spatial Data Handling, ed. P.F. Fisher. Springer, Berlin, Heidelberg, 327-342.

Bertin J. 1983. Semiology of graphics: diagrams, network, maps. The University of Winsconsin Press, Madison.

Boyce R., Clark W. 1964. The concept of shape in geography. Geographical Review, 54.

Brassel K.E., Weibel R. 1988. A review and conceptual framework of automated map generalization. International Journal of Geographical Information Science, 2, 3, 229-244.

Cebrykow P. 2017. Cartographic generalization yesterday and today. Polish Cartographical Review, 49, 1, 5-15. DOI: 10.1515/pcr-2017-0001

Chaudhuri D., Kushwaha N.K., Sharif I., Samal A. 2012. Finding best-fitted rectangle for regions using a bisection method. Machine Vision and Applications, 23, 6.

Chrobak T. 2010. The role of least image dimensions in generalized of object in spatial databases. Geodesy and Cartography, 59(2), 99-120.

Chrobak T., Keller S.F., Kozioł K., Szostak M., Żukowska M. 2007. Podstawy cyfrowej generalizacji kartograficznej, wyd. 1. Uczelniane Wydawnictwa Naukowo-Dydaktyczne AGH, Kraków.

Dent B.D. 1996. Cartography: Thematic Map Design. 5th ed. Dubuque, C. Brown Publishers.

Douglas D.H., Peucker T.K. 1973. Algorithms for the Reduction of the Number of Points Required to Represent a Digitised Line or its Caricature. Cartographica: The International Journal for Geographic Information and Geovisualization, 10(2).

Harrie L., Weibel R. 2007. Modelling the Overall Process of Generalisation. In: Generalisation of Geographic Information: Cartographic Modelling and Applications, eds. W. Mackaness, A. Ruas, L. Sarjakoski. T. ICA, Elsevier.

He X., Zhang X., Yang J. 2018. Progressive Amalgamation of Building Clusters for Map Generalization Based on Scaling Subgroups. ISPRS Int. J. Geo-Inf., 7, 116.

Hu M.K. 1962. Visual pattern recognition by moment invariants, computer methods in image analysis. IRE Transactions on Information Theory, 8.

Kohl J.G. 1850. Der Verkehr und die Aussiedlungen der Menschen in ihrer Abhängigkeit von der Gestaltung der Erdoberfläche. Leipzig.

Kosturbiec B. 1972. Analiza zjawisk koncentracji w sieci osadniczej. Prace Geograficzne, 93. Instytut Geografii Polskiej Akademii Nauk, Wydawnictwo Polskiej Akademii Nauk.

Kwinta A., Gniadek J. 2017. The description of parcel geometry and its application in terms of land consolidation planning. Computers and Electronics in Agriculture, 136. 
Liu W., Zhang X., Li S., Ying M. 2010. Reasoning about cardinal directions between extended objects. Artificial Intelligence, 174, 12-13.

Loy G., Barnes N., Shaw D., Robles-Kelly A. 2005. Regular polygon detection. Proceedings of the 10th IEEE International Conference on Computer Vision, 1.

Maceachren A.M. 1985. Compactness of geographic shape: Comparison and evaluation of measures. Geografiska Annaler, 67B.

Mackaness W.A., Ruas A. 2007. Evaluation in the Map Generalisation Process. In: Generalisation of Geographic Information: Cartographic Modelling and Applications, eds. W.A. Mackaness, A. Ruas, L.T. Sarjakoski. Elsevier, Amsterdam, 89-111.

Molano R., Rodríguez P.G., Caro A., Durán M.L. 2012. Finding the largest area rectangle of arbitrary orientation in a closed contour. Applied Mathematics and Computation, 218, 19.

Müller J.C., Lagrange J.P., Weibel R. 1995. GIS and generalization. Methodology and Practice. Taylor and Francis, London.

Peura M., Iivarinen J. 1997. Efficiency of Simple Shape Descriptors. 3rd International Workshop on Visual Form, Capri, Italy.

Pilkey W.D. 1994. Formulas for Stress, Strain, and Structural Matrices. John Wiley \& Sons, Inc., New York.

Prokop J., Reeves A.P. 1992. A survey of moment-based techniques for unoccluded object representation and recognition. Computer Vision, Graphics and Image Processing, 54, 5.

Sarkar A., Biswas A., Dutt M., Bhattacharya A. 2018. Finding a largest rectangle inside a digital object and rectangularization. Journal of Computer and System Sciences, 95.

Smith J.R., Chang S.F. 1996. VisualSEEk: a fully automated content-based image query system. Proceedings of the Fourth ACM International Conference on Multimedia, 18-22 November 1996, Boston, Massachusetts, USA.

Stojmenović M., Žunić J. 2008. Measuring elongation from shape boundary. Journal of Mathematical Imaging and Vision, 30.

Williams E.A., Wentz E.A. 2008. Pattern analysis based on type, orientation, size and shape. Geographical Analysis, 40.

Zandonadi R.S., Luck J.D., Stombaugh T.S., Shearer S.A. 2013. Evaluating field shape descriptors for estimating off-target application area in agricultural fields. Computers and Electronics in Agriculture, 96.

Zunic J., Rosin P. 2004. A new convexity measure for polygons. IEEE Trans. Pat. Ana. \& Mach. Int., $26,7$.

Dr hab. inż. Andrzej Kwinta, prof. UR

Uniwersytet Rolniczy w Krakowie

Katedra Geodezji

ul. Balicka 253a, 30-147 Kraków

e-mail: andrzej.kwinta@urk.edu

ORCID: 0000-0002-2003-7703

Dr hab. inż. Joanna Bac-Bronowicz, prof. PWr

Politechnika Wrocławska

Katedra Geotechniki, Hydrotechniki, Budownictwa Podziemnego i Wodnego

Plac Grunwaldzki 9, 50-377 Wrocław

e-mail: joanna.bac-bronowicz@pwr.edu.pl

ORCID: 0000-0001-9038-1131 